\title{
A propos de l'usage du champ magnétique terrestre pour l'étude des rayons eosmiques ou de l'activité magnétique
}

\author{
PIERRE-NOËL MAYAUD
}

Depuis plusieurs années déjà, on a cherché à déterminer expérimentalement la position du minimum d'intensité de la composante nucléaire en fonction de la latitude, et rapidement des écarts considérables, variant d'ailleurs avec la longitude, ont été constatés entre cette position et l'équateur géomagnétique, alors que la théorie prévoit leur coïncidence. Des hypothèses diverses $\left(^{1}\right)$ ont été avancées pour rendre compte de tels écarts; mais derniérement, P. Rothwell a montré qu'ils coïncidaient - au moins dans les régions africaines - avec les anomalies du champ magnétique terrestre $\left({ }^{2}\right)$.

Or nous avions eu l'occasion ( $\left.{ }^{3}\right)\left({ }^{4}\right)$ d'attirer l'attention des géomagnéticiens sur le fait que le champ géomagnétique, actuellement utilisé pour ordonner les phénomènes magnétiques observés, était sans doute inapte á remplir ce rôle. Certes, il avait été légitime d'abandonner les valeurs au sol pour un tel usage devant l'évidence que les corpuscules responsables des phénomènes ne subissent pas l'influence de ce champ au sol, souvent fortement déformé par des anomalies superficielles; mais n'était-ce pas tomber dans l'excès inverse que de lui substituer le champ géomagnétique, pure abstraction mathématique ne conservant de l'analyse sphérique harmonique des données d'observation que l'unique premier terme, alors que, seule, la fonction mathématique comprenant tous les termes de cette analyse pouvait prétendre décrire de manière approchée mais significative la réalité physique visée? De ceci, une preuve en quelque sorte mathématique est donnée par les résultats de l'ana- lyse qui montrent que l'importance relative, par rapport au premier terme, des autres termes est de $14.7 \%$ au sol, $5.4 \%$ à 6.300 $\mathrm{km}$ d'altitude, $1.8 \%$ à $25.000 \mathrm{~km}$ et $0.9 \%$ à $57.000 \mathrm{~km}$ : le champ géomagnétique ne commence donc à bien représenter le champ réel qu'au delà de $30.000 \mathrm{~km}\left({ }^{*}\right)$; aux altitudes inférieures, il ne peut en fait pas plus prétendre que les valeurs magnétiques au sol ordonner effectivement les phénomènes observés, et c'est pourtant peut-être à ces altitudes, où l'intensité du champ commence à devenir beaucoup plus forte, que la forme réelle du champ aura toute son importance dans l'étude des phénomènes. De cette conclusion toute théorique, une preuve expérimentale était tentée en montrant que les coordonnées magnétiques (inclinaison et déclinaison) qui ordonnaient le mieux les phénomènes d'activité magnétique - ce-là qui sont la manifestation la plus directe d'une cause corpuscolaire - étaient représentées par la configuration réelle du champ vers 3.000 à $5.000 \mathrm{~km}$ d'altitude, telle qu'ellc peut être déduite de l'analyse de E. H. Vestine $\left({ }^{5}\right)$. La signification d'un tel résultat risque cependant d'être pleine d'équivoques, et sans doute ne les avions-nous pas entiérement dissipées dans la présentation que nous en avions faite ([3] p. 91, [4], p. 51);

(*) Les $5 \%$ des $6.300 \mathrm{~km}$ causent des déformations parfois considérables des isoclines; ainsi, dans les régions sibériennes, les courbes qui représentent la projection sur la surface de la terre de ces isoclines sont distantes de $600 \mathrm{~km}$ des courbes représentant les isoclines géomagnétiques correspondantes. 
c'est pourquoi nous essaierons à nouveau d'exposer cette signification $\left({ }^{*}\right)$.

Nous noterons d'abord que, si les géomagnéticiens - et après eux les cosmiciens ont choisi le champ géomagnétique pour ordonner les phénomènes, c'est qu'ils se trouvaient devant un problème tridimensionnél, à savoir le mouvement des corpuscules dans un espace à trois dimensions où existe un champ magnétique qui lui aussi est tridimensionnel, et que ce champ " géomagnétique " leur était un moyen commode de décrire le champ tridimensionnel de la terre. L'idéal serait de prendre le champ magnétique tridimensionnel tel qu'il peut être déduit en tout point de l'espace à partir de l'analyse sphérique. Mais à qui connaît d'une part la complexité du calcul des trajectoires dans le champ d'un dipôle, il est évident qu'une telle solution apparaît impossible à retenir pour les études théoriques; et d'autre part comment ordonner les observations faites à la surface de la terre (espace à deux dimensions) par une quantité tridimensionnelle (le champ magnétique réel) $\left({ }^{* *}\right)$ ? Sans doute est-il justifié d'utiliser le champ géomagnétique pour des calculs théoriques, $\dot{a}$ condition toutefois de ne pas vouloir chercher ensuite une coïncidence précise entre prévisions ainsi obtenues et observations: une telle coïncidence ne peut être puisque le champ réel n'est pas le champ géomagnétique. Mais une telle simplification n'étant pas possible pour ordonner les observations elles-mêmes, il deviendra nécessaire de s'orienter vers une solution intérmédiaire.

Pour en comprendre la portée, nous ferons d'abord une remarque de vocabulaire: l'expression " champ au sol " - comme celle de " champ à une altitude donnée " - est équivoque, car ce "champ aux sul" n'est en fait qu'une coupe à deux dimensions dans le champ réel qui, lui, est tridimensionnel

(*) Nons voudrions ici remercier Mr. F. Thellier, qui nons a aidé par ses critiques à clarifier notre pensée.

$\left.{ }^{* *}\right)$ Bien que le champ géomagnétique soit lui aussi tridimensiomnel, on ne se heurte pas à la même difficulté dans son utilisation; par exemple, sur la verticale d'un lieu de la surface de la terre, inclinaison et léclinaison sont constantes, elles ne le sont pas dans le cas du champ réel. comme tout champ $\left({ }^{*}\right)$; et pourrait-on ajouter, le champ géomagnétique, considéré vis à vis de la réalité physique existante n'est lui-même qu'une telle coupe, c'est à dire que, à parler en toute rigueur, il ne représente le champ réel que sur la surface de la sphère de rayon infini, concentrique à. la terre. Par conséquent, aucun de tous ces champs - au sol, à une altitude quelconque, ou géomagnétique - ne peut suffir à ordonner les phénomènes observés, bien que chacun d'eux represente le champ réel sur la surface d'une sphère donnée, ceci à l'approximation près de l'analyse sphérique (*). Il a été commode, et ce n'était rien de plus que cela, d'utiliser la "coupe " représente par le champ au sol, puis on lui a substitué celle que représente le champ géomagnétique; mais rette dernière solution n'est plus suffisante à cause de la finesse plus grande atteinte par les observations, et il devient necessaire d'utiliser une "coupe " intermédiaire entre le champ au sol et le champ géomagnétique. Le critère qui peut permettre de faire le choix de cette coupe iner'médiaire est simple; ainsi, lorsque la theorie montre quc dans le champ d'un dipôle tel phénomène devrait avoir une intensité constante à une latitude donnée, la "coupe " la meilleure sera celle qui manifestera le mieux cette constance, et on sera alors conduit pour ordonner le phénomène à utiliser les "valeurs magnétique " à une altitude donnée.

Certes il ne se passe rien de spécial à une telle altitude; la seule róalité physique agissante est le champ réel dans tout l'espace, et la coupe ainsi faite n'en donne qu'une image conventionnelle. On peut dire d'elle qu'elle est l'image la plus commode pour ordonner les phénomènes, et l'avantage certain qu'elle procure est de supprimer des

(*) Il faudrait employer pour etre exact l'expression "valeur magnétique ", et non pas "champ ".

(**) On notera que cette approximation n'est pas moins bonne que celle-là même qui existe pour le champ géomagnétique puisque l'une et l'autre sont celles de la mếme analyse sphérique harmonique des mêmes données expérimentales. Par contre, ntiliser le champ géomagnétique aillenrs qu'à l'infini ou à de grandes distances de la terre est introduire une approximation supplénıentaire. 
faux problèmes provoqués par l'utilisation du champ géomagnétique; ou, tout au moins faudrait-il, avant de chercher des explications aux écarts observés entre observations et champ géomałnétique, voir si ces écarts ne peuvent être réduits par l'emploi d'une autre "coupe " - le champ géomagnétique, nous l'avons dit, n'est en fait au plan des observations et de la réalité q'une "coupe " parmi d'autres - plus représentative du champ réel agissant.

La complexité ainsi introduite dans l'étude des phénomènes qui dépendent du champ magnétique terrestre est évidente; car chaque observatoire ou lieu d'observation devrait se voir attribuer des inclinaisons magnétiques multiples selon le type de phénomène; et, de plus, il faudrait tenir compte de la variation séculaire. Mais aussi longtemps que l'on refusera une telle complexité, il semble que l'on se heurtera inévitablement à des eflets parasites dans l'interprétation des phénomènes, et ceci inutilement.

Les conclusions prórédentes avaient été obtenues à partir de resultats expérimentaux provenant des seules régions de haute latitude; c'est là seulement en effet qu'arrivent les corpuscules responsables des variations rapides du champ magnétique observées à la susface da la terre. Les rayons cosmiques permettent, semble-t-il, de confirmer de telles conclusions en montrant leur validité pour les régions de latitude moyenne ou équatoriale.

Pour cela, nous utiliserons les mesures mentionnées ci-dessus $\left({ }^{2}\right)$ et reprendrons d'autres mesures $\left({ }^{1}\right)\left({ }^{8}\right)$. Dans l'utilisation de telles données, qui souvent ont été entreprises surtout pour déterminer l'équateur des rayons cosmiques, nous ne chercherons pas à déterminer en lui-même un tel équateur; en effet déterminer l'abscisse d'un minimum sur une courbe expérimentale est une opération sujette à trop d'erreurs. Mais, donnant la même importance aux mesures faites à toute latitude, nous chercherons le système de coordonnées magnétiques, e'està-dire encore l'altitude à laquelle les valeurs magnétiques réelles permettent d'obtenir la meilleure symétrie entre les deux hémisphères pour l'intensité des rayons cosmiques en fonction de la latitude, ou encore la meilleure homogénéité entre différents méridiens pour un même hémisphère, en partant dı principe qu'avant tout antre cause de variaiion de ce plićnomène, à lriitude magnétique égale doit correspondre intensite égale. Nous emploierons systématiquement comme abscisse la latitude magnétique au lieu de l'inclinaison bien que la notion de latitude ne puisse en fait être définie que pour le champ du dipôle; une telle liberté est permise en tant que la relation mathématique ( $\operatorname{tg} 1=2 \operatorname{tg} \Phi)$ qui lie les deux quantités dans le champ d'un dipôle et que nous conservons arbitrairement ici est une fonction univoque et continue; et, parce que cette "latitude " a l'avantage de fournir un paramètre d'espace qui est grossièrement en relation linéaire avec les distances le long des méridiens, ce que ne fait pas l'inclinaison on évite une concentration des points daus les régions polaires et leur dispersion dans les régions équatoriales. Les latitudes magnétiques à claque altitude ont été déduites des données de E. H. Vestine $\left(^{5}\right)$.

La figure 1 représente la variation de l'intensité de la composante nucléaire en fonction de la latitude magnétique au sol, à $300 \mathrm{~km}$, ̀̀ $1.000 \mathrm{~km}, 5.000 \mathrm{~km}$ d'altitude et du dipôle. Les échelles d'intensité qui varient avec chacun des ensembles de mesures sont indiquées. Les cercles correspondent aux latitudes Nord, les croix aux latitudes Sud.

A) Les données de D. C. Rose (1), recueillies lor des voyages du "Labrador " autour de l'Amérique de Nord et de " l'Atka " dans l'Antarctique avec aller par le Pacifique et retour par l'Atlantique, ont élé utilisées de la manière suivante: 1) étant donné le très grand nombre de points dont chacun correspond à douze heures d'observation, on a fait la moyenne des intensités moyennes observées dans des aires gcographiques limitées en gros par des méridiens de 4 degrés en 4 degrés et res parallèles de 2 degrés en 2 degrés; 2) pour les v: leurs ainsi obtenues, on a mesuré la latitude magnétique aux différentes altitudes ou au sol, corres pondant au barycentre de chacun des groupes de points contenus dans les aires précédentes; 3 ) on a fait alors à nouveau la moyenne des valeurs correspondant à des tranches de latitudes magnétiques allant de 5 en 5 de- 
grés; 4) pour les latitudes $20^{\circ} \mathrm{N}$ à $50^{\circ} \mathrm{N}$, les écarts à la moyenne des intensités ont été calculés et sont représentés sur les graphiques par des barres proportionnelles; cette tranche de latitude représente trois passages dans l'Atlantique Nord et un passage dans le Pacifique Nord; 5) les courbes tracées l'ont été à partir des seuls points de l'hémisphère Nord; 6) entre les latitudes $10^{\circ} \mathrm{N}$ et $50^{\circ} \mathrm{S}$, on a porté les points individuels obtenus en 2 ); ces points représentent les deux passages de "l'Atka " à ces latitudes (le trajet retour par l'Atlantique est forme par l'ensemble de points - petites croix - qui sont très au-dessus de la courbe moyenne dans le cas du champ au sol, à $300 \mathrm{~km}$ et à $1.000 \mathrm{~km}$ ).

B) Pour les données P. Rothwell $\left({ }^{2}\right)$, on a mesuré la latitude magnétique aux différentes altitudes on au sol pour chaque point - chacun d'eux correspond à six heures d'observation -, puis l'opération 3) précédente a été effectuée. Les données utilisées correspondent à deux voyages successifs du "Roxburgh" dans le sens Sud-Nord de Cape Town à Southampton par la côte Ouest de l'Afrique. Chaque point du graphique est la moyenne de cinq à dix points observés. Les courbes tracées l'ont été à partir des seuls points de l'hémisphère Nord.

C) Pour les données M. Oda, la latitude magnétique aux différentes altitudes ou au sol a été mesurée pour chaque point chacun d'eux correspond à 24 heures d'observation - Pour les point Sud dont la latitude est supérieure à $50^{\circ}$, de même que pour tous les points Nord, on a porté sur le graphique les points individuels; pour les points Sud dont la latitude est comprise entre 0 et $50^{\circ}$, on a effectué l'opération 3 ). Ces données correspondent géographiquement à un trajet du "Soja " depuis l'équateur vers $100^{\circ} \mathrm{E}$ de longitude jusqu'au cercle polaire Antarctique vers $70^{\circ} \mathrm{E}$ en passant par Cape Town et retour à peu près par la même voie jusqu'au Japon via Singapour. Sur ce graphique, chaque point correspondant à une moyenne de mesures est ordinairement la moyenne de 3 à 5 mesures individuelles, parfois beaucoup plus. Les courbes tracées l'ont été à partir des seuls points de l'hémisphère Sud.
Sur catte figure, on jeut faire les remarques suivantes. Les mesures $A$, aux latitudes moyennes Nord, se regroupent le mieux, selon les écarts à la moyenne figurés pour chaque cercle, pour 1.000 ou $300 \mathrm{~km}$ d'altitude; tandis que los mesures Sud de l'aller de l' "Atka " restent toujours à l'intérieur des marges d'écart par rapport ì la courbe movenne Nord, l'anomalie dans l'intensité des ravons cosmiques, qui apparaît aux latitudes équatoriales dans le cas du champ du dipôle pour les mesures du retour de l'"Atka \#, se développe et semble exister aussi aux latitudes moyennes Sud. Les mesures $B$ apparaissént être le mieux coordonnées quant à la comparaison des deux hémisphères vers $1.000 \mathrm{~km}$ d'altitude; l'écart qui subsiste alors entre $10^{\circ}$ et $20^{\circ}$ est certainement beaucoup moins important que celui qui apparaît avec le champ au sol entre $20^{\circ}$ et $50^{\circ}$; la question resterait posée de savoir si l'altitude de meilleur coordination varierait avec la latitude, mais de nouvelles mesures seraient sans doute nécessaires pour répondre à cette nouvelle question. Dans les mesures $C$, les points Nord restent systématiquement au-ressus des points Sud, l'écart étant cependant le plus faible dans le cas du champ à 1.000 ou $5.000 \mathrm{~km}$. Ce qui est sans doute le plus remarquable est que avec le champ du dipôle, l'inflexion (ou le maximum) de la courbe tracée au travers des valeurs Sud s'amorce dès $35^{\circ}$ de latitude; et ceci entraînerait évidemment une différence considérable d'avec les mesures $A$ et $B$, à supposer que, après normalisation des intensités, on cherche à faire une superposition des courbes $\mathrm{A}, \mathrm{B}$ et $\mathrm{C}$ pour les comparer; de ce point de vue, les courbes $C$ pour $1.000,300 \mathrm{~km}$ ou au sol apparaissent beaucoup plus normales. Enfin, la dispersion des points au-dessus des latitudes $50^{\circ} \mathrm{S}$ correspond à une aire géographique relativement restreinte; elle semble donc due plus aux rayons cosmiques eux-mêmes plutôt qu'à des anomalies magnétiques.

En conclusion, les trois séries de mesures ici étudiées semblent bien confirmer la conclusion obtenue antérieurement pour les seules hautes latitudes et pour un autre type de rayonnement corpusculaire: le champ du dipôle s'avère incapable d'ordonner, aux latitudes moyennes ou équatoriales, les in- 


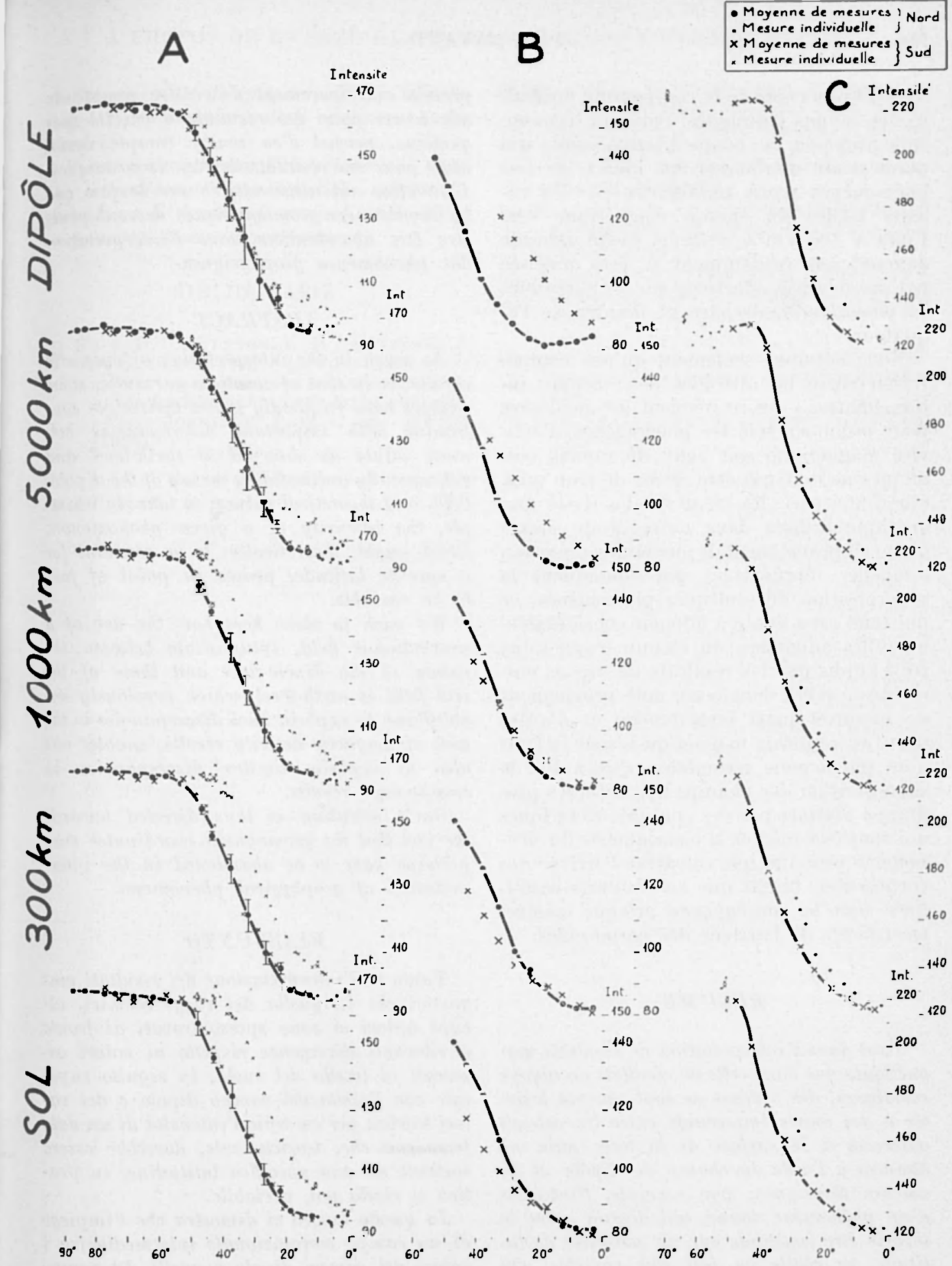

Fig. 1 - Variation en fonction de la latitude magnétique au sol, à diverses altitudes ou géomagnétique de l'intensité de la composante nucléaire des rayons cosmiques observée sur differentes routes maritimes; pour obtenir le nombre d'impulsions enregistrées par heure, il faut respectivement multiplier les échelles d'intensité indiquées sur les différents graphique par 64 (A), $100(\mathrm{~B})$ et 32 (C). 
tensités observées de la composante nucléaire des rayons cosmiques. Selon la terminologie proposée, la " coupe intermédiaire " qui permettrait d'ordonner au mieux de tels phénomènes serait représentée par les valeurs réelles du champ magnétique vers 1.000 à $300 \mathrm{~km}$ d'altitude. Cette altitude demanderait évidemment à être précisée par des mesures effectuées sur les longitudes les plus diverses de part et d'autre de l'équateur.

Nous noterons seulement qu'une comparaison entre les altitudes des "coupes intermédiaires " qui se révèlent les meilleures pour ordonner soit les phénomènes d'activité magnétique soit ceux de rayons cosmiques ne doit pas être serrée de trop près. Car d'une part, les résultats d'activité maynétique utilisés dans rotre étude précédente $\left({ }^{3}\right)$ proviennent de moyennes annuclles, lesquelles représentent par conséquent la superposition de multiples phénomènes, ce qui tend sans doute à adoncir considérablement les anomalies du champ magnétique réel, tandis que les résultats de rayons cosmiques étudiés dans cette note proviennent de mesures quasi instantanées; et d'autre part, les résultats magnètiques sont le fruit d'un phénomène secondaire, c'est-à-dire la superposition des champs magnétiques produits à distance par des courants électriques qui sont eux-mêmes la conséquence du phénomène primaire que constitue l'arrivée des corpuscules, tandis que les résultats cosmiques sont la conséquence presque uniquement locale de l'arrivée des corpuscules.

\section{RESUME}

Tant dans l'interpretation de résultats $m a-$ gnétiques que dans celle de résultats de rayons cosmiques, des auteurs se sont souvent heurtés à des écarts importants entre les valeurs observées à la surface de la terre puis ordonnées à l'aide du champ du dipôle et les valeurs théoriques; par exemple, l'intensité d'un phénomène donne, qui devrait selon la théorie être constante sur un parallèle de latitude, se révèle en fait être variable. On montre ici que l'emploi d'un champ conventionnel, intermédiaire entre le champ du dipôle et le champ au sol, emploi qui avait permis antérieurement de rendre compte de tels écarts pour des résultats d'activité magnétique, permet d'en rendre compte également pour des résultats de rayons cosmiques. L'attention est ainsi attirée sur le fait que les coordonnées géomagnétiques devront peutêtre être abandonnées dans l'interpretation des phenomènes géophysiques.

\section{$A B S T R A C T$}

As much in the interpretation of magnetic results as in that of cosmicrays results, some authors have frequently found theirselves confronted with important discrepancies between values as observed at earth-level and subsequently ordinated by means of the dipolefield, and theoretical values; to take an example, the intensity of a given phenomenon, which ought, theoretically, to be constant for a specific latitude, proves in point of fact to be variable.

We wish to show here how the use of a conventional field, intumediate between the values of the dipole-field and these of the real field at earth-level, which previously enabled one to explain such discrepancies in the case of magnetic activity results, enables one also to explain similar discrepancies in cosmic-rays results.

One's attention is thus directed towards the fact that the geomagnetic coordinates may perhaps have to be abandoned in the interpretation of geophysical phenomena.

\section{RIASSUNTO}

Tanto nell'interpretazione dei risultati magnetici che in quella dei raggi cosmici, alcuni autori si sono spesso trovati di fronte a rilevanti divergenze rispetto ai valori osservati al livello del suolo, in seguito superate con l'aiuto del campo dipolo e dei valori teorici; per esempio l'intensità di un dato fenomeno che, teoricamente, dovrebbe essere costante su una specifica latitudine, in pratica si rivela poi, variabile.

In questo lavoro si dimostra che l'impiego di un campo convenzionale intermedio tra $i$ valori del campo dipolo e quelli del campo reale al livello del suolo, impiego che aveva permesso, in precedenza, di spiegare tali divergenze nel caso di risultati di attivïtà ma- 
gnetica, permette di spiegare, altresi, tali divergenze per alcuni risultati relativi ai raggi cosmici.

Viene cosi attirata l'attenzione sul fatto che le coordinate geomagnetiche, nell'interpretazione di fenomeni geofisici, dovrebbero - forse - essere abbandonate.

\section{BIBLIOGRAFIE}

(1) Rose, D. C.; Fenton, K. B.; Katztyan J. et SIMPson, J. A., Latitude effect of the cosmic ray ucleon and meson components at sea level from the Arctic to the Antarctic, "Can. Journ. of Phys, ", 34, 968-984 (1956).
(2) Rotiwell, P. et Queniy, J., Report at Varenna Conference, June 1957, to be published in "Nuovo Cimento ", (le detail des mesures utilisées dans cette note nous a été directement communiqué par Miss P. Rothwell).

$\left({ }^{3}\right)$ Ma Y aud, I. N., Activité magnétique dans les régions polaires, "Ann. Geophys. ", 12, 84.100 (1956).

(4) - Terre Adélie 1951-1952, "Magnetisme terrestre ", fasc. Il, Activité magnétique dans les régions polaires, Expéditions $\mathrm{P}^{\mathrm{B}}$ laires Francaises, Paris, 1955.

(5) Vestine, E. H., The Geomagnetic Field, Carnegie Institution, 1947, Publication 580.

(6) ODA, M., Report al Varenna Conference, June 1957. 